\title{
CMEARTICLE Primary care approach to Kawasaki disease
}

Joanne Mui Ching $\underline{T a n}^{1}$, MMed, MRCPCH, Jeanette Keng Wein $\underline{\operatorname{Tan}}^{1}$, MMed, MRCPCH, Choon How $\underline{H o w}^{2}$, MMed, FCFP, Kai Liang ${\underline{T e h^{3}}}^{\text {, MMed, MRCPCH }}$

His mother took Tom, a one-year-old boy, to your clinic for a repeat consultation for persistent fever. Two days ago, he had presented with a three-day history of high fever and conjunctivitis. His mother mentioned that he had started attending a neighbourhood childcare centre one month before, and his teachers had said that a few of his classmates were down with flu. At that time, you prescribed antipyretics for viral fever and advised his mother to return if the fever persisted beyond Day 5 of the illness. During the repeat consultation, Tom's mother reported that he had developed a rash two days ago, but it had mostly faded. She showed you a photo she had taken with her mobile phone of a generalised maculopapular rash. Tom was extremely fretful and refused to lie down on the couch, but you managed to examine him on his mother's lap. On examination, his neck was supple, and a detailed head-to-toe examination revealed bilateral non-suppurative conjunctivitis, mild red lips and Bacille Calmette-Guérin vaccine site erythema. You were concerned that Tom had features of Kawasaki disease and referred him to a paediatric tertiary hospital for further evaluation.

\section{WHAT IS KAWASAKI DISEASE?}

Kawasaki disease $(K D)$ is an acute, self-limiting febrile illness that predominantly affects young children below five years of age. ${ }^{(1)}$ It is one of the most common vasculitis disorders of childhood and, if left untreated, is associated with development of coronary artery aneurysms (CAA). It is now the most common cause of acquired heart disease in children in developed countries. ${ }^{(2)}$ The aetiology of KD remains unknown. A variety of infectious agents such as retrovirus, Epstein-Barr virus, coronavirus, staphylococcal and streptococcal superantigens, and unidentified virus particles have been postulated to trigger an immune activation, driving the inflammatory process. ${ }^{(3,4)}$ To date, no single agent has been consistently identified.

\section{HOW RELEVANT IS THIS TO MY PRACTICE?}

Primary care physicians play a crucial role in the early diagnosis of KD, as they are often the first contact for patients. Many patients with KD initially present to primary care clinics for acute undifferentiated fever, and the attending doctor has the duty of diagnosing KD. Very often, the diagnosis is missed due to the mindset that KD is 'rare' and will not be seen in general practice. In reality, the incidence of KD is increasing, and the prevalence is higher among Asian populations. The incidence of KD in Singapore, estimated at 51.4 per 10,000 children aged below five years, is one of the highest outside Japan and very similar to that in Hong Kong and Taiwan. ${ }^{(5)} \mathrm{KD}$ results in CAA in up to one-third of untreated patients, and persistent aneurysms in $3 \%-5 \%$ of patients who receive treatment, ${ }^{(6,7)}$ putting them at lifelong risk of thrombosis and myocardial ischaemia. Patients aged below six months or above five years are at increased risk for CAA. A recent study by Tulloh et al in the United Kingdom (UK) found that children with KD treated after Day 7 of illness were at significantly increased risk of serious heart damage, and this risk rose proportionately with further treatment delay ${ }^{(8)}$ Similarly, the American Academy of Paediatrics suggested that treatment should ideally be administered within the first seven days of illness and by Day 10 (as defined by the first day of fever) at the latest. ${ }^{(9)}$ The American Heart Association (AHA) recommended that intravenous immunoglobulin (IVIG) should be instituted as early as possible, within the first ten days of illness of the onset of fever, as soon as the diagnosis can be established. ${ }^{(10)}$ This further highlights the pivotal role of primary care providers in early diagnosis to ensure that patients receive prompt treatment for KD.

There is no single diagnostic test for KD. Clinical criteria recommended by the AHA are used to diagnose KD (Box 1). ${ }^{(10)}$ A study conducted in the UK by Moore et al reported that most children who presented initially to primary care did not fulfil the criteria for complete KD. Fever and polymorphous rash were the most common initial presenting features. These children developed more signs during their course of admission. Most general practitioners (GPs) did not consider KD in their differential diagnosis. ${ }^{(11)}$ This study highlighted the diagnostic challenge of KD and the importance of keeping KD in mind when children present with fever and rash. We hope that with greater awareness of this condition, there will be earlier recognition and a higher index of suspicion of KD in children with non-specific febrile illness.

Since April 2020, there have also been reports of COVID-19 infection with multi-systemic inflammatory syndrome that shares many characteristics with $\mathrm{KD}$. This syndrome is now known as

${ }^{1}$ Department of Paediatrics, KK Women's and Children's Hospital, ${ }^{2}$ Care and Health Integration, Changi General Hospital, ${ }^{3}$ Department of Immunology and Rheumatology, KK Women's and Children's Hospital, Singapore

Correspondence: Dr Joanne Tan Mui Ching, Department of Paediatrics, KK Women's and Children's Hospital, 100 Bukit Timah Rd, Singapore 229899. joanne.tan.m.c@singhealth.com.sg 
multi-system inflammatory syndrome in children (MIS-C). It was first reported by Riphagen et al in the UK in late April, with an unprecedented cluster of eight children with hyperinflammatory shock, showing features similar to KD. ${ }^{(12)}$ By mid-May, the New York City Health Department detected 89 paediatric patients with symptoms consistent with MIS-C.(13) Locally, there have been no reported cases of MIS-C or KD linked to COVID-19 so far. The Ministry of Health in Singapore has urged for increased vigilance and referral of potential cases that present with clinical features suggestive of KD to tertiary paediatric hospitals for evaluation.

A high index of suspicion is required for the diagnosis of incomplete KD. Incomplete KD should be considered in any infant or child with prolonged unexplained fever and fewer than four of the principal clinical findings. In these cases, further laboratory investigations and echocardiography may be required to support the diagnosis of incomplete KD.

\section{WHAT CAN I DO IN MY PRACTICE?}

Acute undifferentiated fever in children is one of the most frequent presenting complaints in primary care. The common diagnosis is infection, with viral infections being more common than bacterial infections. The younger the child is, the higher the probability of serious bacterial infection. Febrile neonates and infants below three months of age are considered a separate high-risk group due to the risk of late-onset Group B streptococcal infection and should be referred to a tertiary paediatric hospital for further management. In a non-verbal child, it is important to conduct a head-to-toe physical examination, actively looking out for 'hidden' sources of infection such as meningitis, otitis media, pharyngitis, septic arthritis, osteomyelitis and urinary tract infection.

Other causes of acute fever include inflammatory or autoimmune causes such as KD and vaccine-related fever. However, vaccine-related fevers usually last less than 48 hours, and there should be a history of recent vaccination. Most benign, self-limiting viral infections do not cause a fever longer than 5-7 days. Fever of longer than five days' duration should be investigated for bacterial infection or other diagnosis such as KD. Primary care physicians can form a 'safety net' to ensure that the diagnosis of KD is not missed by giving appropriate return advice to parents, such as in the event of persistent fever or clinical features suggestive of KD.

\section{HOW DO I DIAGNOSE KAWASAKI DISEASE?}

Patients with principal clinical findings that meet the case definition by the AHA are said to have classical or complete KD (Box 1). ${ }^{(10)}$ However, KD can present in different spectrums, making diagnosis even more challenging. Some children present with some but not all clinical features of $K D$, known as incomplete $K D$. To aid in the diagnosis of incomplete $\mathrm{KD}$, the AHA proposed an algorithm that includes a constellation of clinical signs and biochemical abnormalities for evaluation of suspected incomplete KD (Fig. 1). ${ }^{(10)}$

Some patients can also present with atypical clinical features such as renal impairment, neuritis, testicular swelling,

\section{Box 1. Clinical criteria to diagnose Kawasaki disease:}

Classical Kawasaki disease is diagnosed in the presence of fever

of $\geq 5$ days, together with at least four of the five principal clinical features:

1. Erythema and cracking of lips, strawberry tongue, and/or erythema of oral and pharyngeal mucosa

2. Bilateral bulbar conjunctival injection without exudate

3. Rash: maculopapular, diffuse erythroderma or erythema multiforme-like

4. Erythema and oedema of the hands and feet in acute phase and/ or periungual desquamation in subacute phase

5. Cervical lymphadenopathy ( $\geq 1.5 \mathrm{~cm}$ in diameter), usually unilateral

pulmonary infiltrates and pleural effusions, diarrhoea, vomiting and abdominal pain, acute surgical abdomen, and haemophagocytic syndrome, which are not commonly seen in KD.

The course of KD can be described in three clinical phases: acute, subacute and convalescent. The acute phase lasts for 7-14 days and is characterised by fever and principal features of KD (Box 1). The subacute phase usually lasts from Day 10 to Day 25 after the onset of illness. During this period, thrombocytosis and desquamation of fingers and toes occur, and CAAs appear. The convalescent phase usually happens 6-10 weeks after onset, when all clinical signs and laboratory abnormalities resolve. Patients with KD typically present to their GP during the acute phase, and early recognition during this phase is crucial to minimise the risk of developing CAA.

$\mathrm{KD}$ is a clinical diagnosis. Taking a detailed history and performing a detailed physical examination with index of suspicion is key in the evaluation of KD. It is also not uncommon for a child to have concomitant infection, such as KD and concurrent upper respiratory tract infection or urinary tract infection. The presence of a differential diagnosis such as infectious mononucleosis or scarlet fever is, very often, then used as evidence to exclude KD as a diagnosis. However, there has been increasing evidence that these infectious agents can act as a trigger for KD. ${ }^{(3,4)}$ Therefore, it is important to bear in mind that the presence of an infectious agent does not exclude the diagnosis of KD.

\section{Obtain history of fever and associated signs and symptoms}

Fever of $\geq 3$ days with at least three features of classical or complete KD, such as rash, conjunctival injection and lymphadenopathy, is sufficient to raise concerns of evolving KD. Signs and symptoms of KD can be transient, and a careful history may reveal that several principal features were present during the illness but resolved by the time of presentation. Not all clinical features present simultaneously. KD features may only start to appear after Day 5 of illness. Similarly, some of the features of KD may be present after fever has lysed without treatment. These groups of patients may still benefit from treatment if there are still signs of ongoing inflammation clinically or serologically. Thus, the presence of any of the five clinical features of KD at any time 


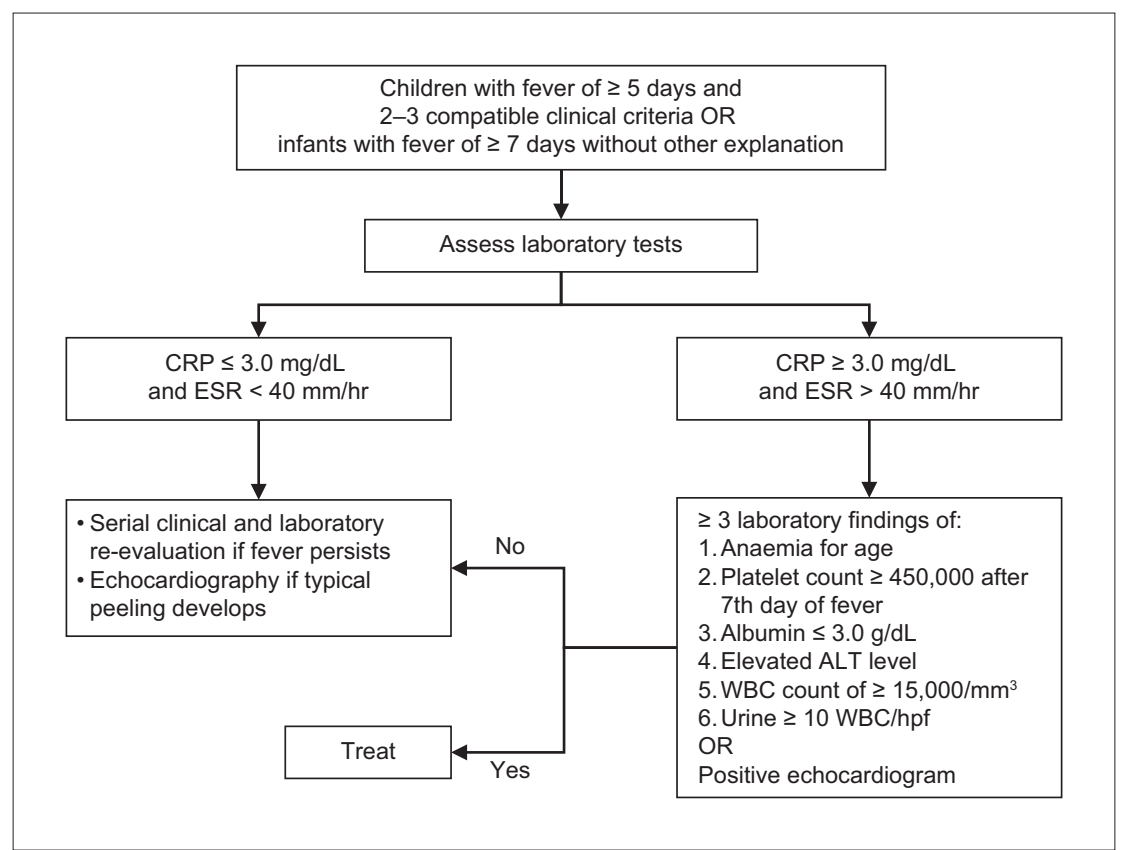

Fig. 1 Flowchart shows evaluation for suspected incomplete Kawasaki disease based on the American Heart Association Guidelines 2017. ALT: alanine aminotransferase; CRP: C-reactive protein; ESR: erythrocyte sedimentation rate; HPF: high-power field; WBC: white blood cell count

during the course of the illness should be documented and taken into consideration.

\section{Examination findings}

Specific signs to look for during an examination include: (1) polymorphous, non-vesicular, non-bullous rash; (2) bilateral painless, non-suppurative, limbic-sparing conjunctivitis; (3) erythema and cracking of lips, strawberry tongue, diffuse injection of oral and pharyngeal mucosa with no discrete lesions or ulceration; (4) significant cervical lymphadenopathy $(>1.5 \mathrm{~cm})$ that is usually unilateral but can be bilateral; and (5) erythema and oedema of hands and feet (Figs. 2a-d). Desquamation of the tips of the fingers and toes (Fig. 2e) and Beau's lines in the nails are often absent during the initial phase of illness and only seen during the convalescent phase. Erythema and induration at the Bacille Calmette-Guérin (BCG) inoculation site (Fig. 2f) is a significant feature of KD in some patients. Thus, the BCG site should be examined carefully in patients with suspicion of KD. Striking irritability is also frequently present. Other significant clinical features include perineal rash and excoriation.

\section{Differential diagnoses to consider}

In patients with exudative conjunctivitis, exudative pharyngitis, ulcerative intraoral lesions, bullous or vesicular rash and generalised adenopathy, the infection - including Group A streptococcal infection, scarlet fever, Ebstein-Barr virus and adenovirus infection - needs to be identified and appropriately treated. Some of these differentials such as Group A streptococcal tonsillitis and scarlet fever can be treated with antibiotics on an outpatient basis; viral infections are usually self-limiting. However, if the fever persists for more than five days or the child does not improve after antibiotic treatment, early referral to the paediatrician is warranted to facilitate further investigations. In patients who present with fever and one or two non-specific features such as rashes, it is important to advise parents to return should the fever persist beyond five days or if the parents notice more features of KD. Other conditions that present in similar ways as KD include drug-related reactions such as Stevens-Johnson syndrome and autoimmune conditions such as systemic juvenile idiopathic arthritis.

\section{WHAT SHOULD I DO IF I SUSPECT KD?}

All patients with suspected KD warrant admission. Box 2 summarises our recommended criteria for referral to a paediatric tertiary hospital. First-line treatments for KD include IVIG and aspirin. Giving IVIG in the acute phase reduces the risk but does not prevent the development of CAAs. In view of the risk of CAA and hypercoagulable state from reactive thrombocytosis, these patients will be started on aspirin to reduce the chance of coronary artery thrombosis. For uncomplicated cases, echocardiography will be done at two weeks, then at 6-8 weeks after the onset of disease to look for coronary artery changes. Aspirin can be discontinued if the patient has no evidence of coronary changes by $6-8$ weeks after the onset of illness. ${ }^{(10)}$ Patients with persistent CAA will require long-term aspirin.

\section{COMMON PITFALLS}

The diagnosis of KD can be easily missed if KD is not considered as a differential diagnosis, especially in patients with incomplete or atypical features. Prolonged fever and irritability may be the only clinical features of KD in an infant below six months of age. The presence of fever and pyuria in an infant or young child can be mistaken for a urinary tract infection, rash may be wrongly diagnosed as allergic rash or viral exanthem, and lymphadenopathy may be overlooked as infective lymphadenitis. 

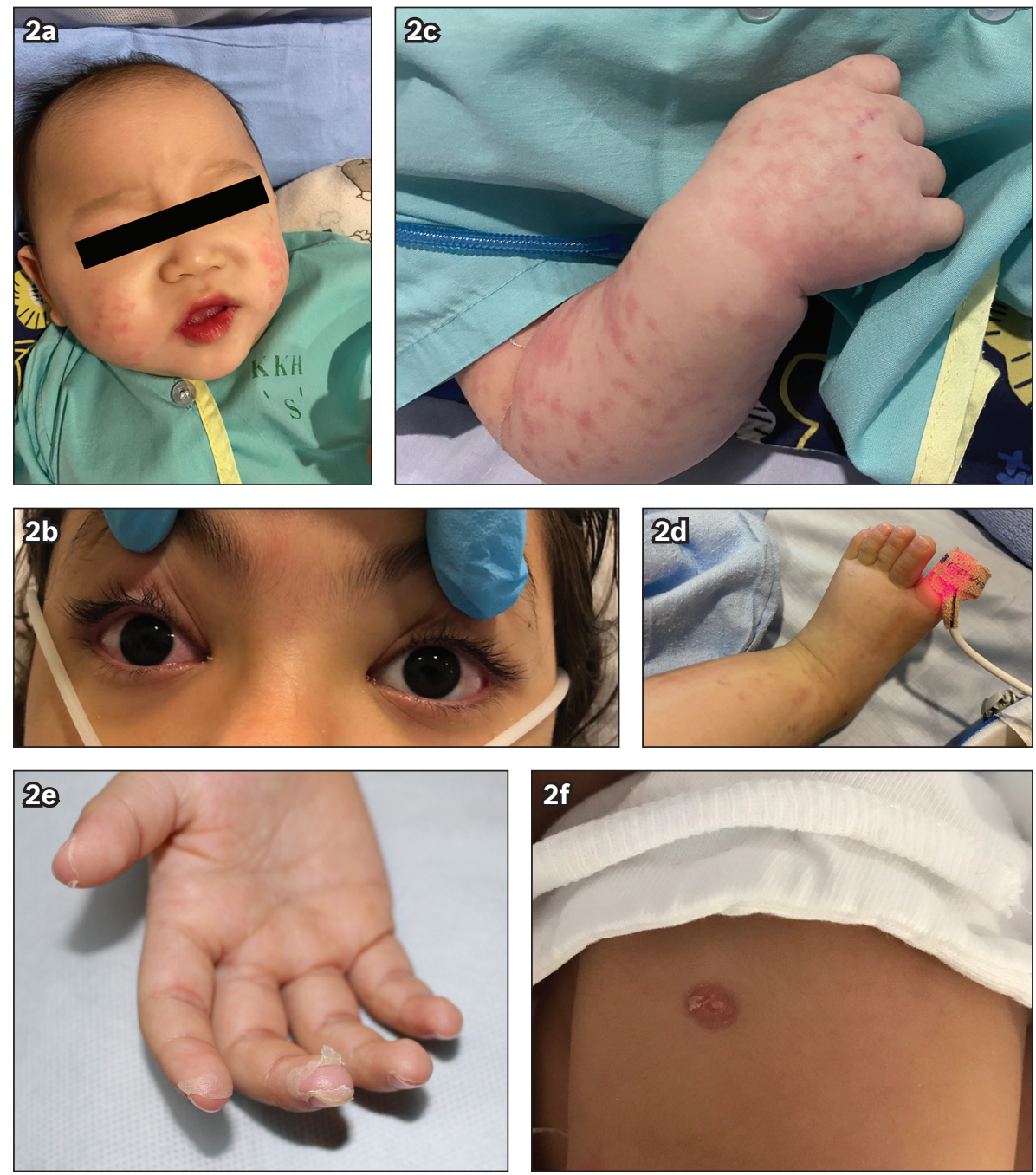

Fig. 2 Photographs show the clinical features of Kawasaki disease: (a) erythema and cracking of lips with maculopapular rash on cheeks; (b) bilateral non-suppurative conjunctivitis; (c \& d) hand and feet oedema with maculopapular rash on arms and legs; (e) desquamation of the fingertips; and (f) Bacille Calmette-Guérin site inflammation.

\section{Box 2. Recommended criteria for referral:}

- Fever $\geq 5$ days with clinical features of Kawasaki disease (KD)

- Fever $\geq 3$ days with $\geq 3$ principal signs of KD

- Fever $\geq 5$ days with no localising source

- Fever with Bacille Calmette-Guérin vaccine site inflammation

- Persistent fever and lymphadenopathy despite appropriate oral antibiotic treatment for $>2$ days

- Febrile urinary tract infection not responding to appropriate oral antibiotic treatment for $>2$ days, especially in patients with negative urine culture

\section{Children with recent KD}

Some children may present at consultations for peeling of skin on the fingertips and toes. In such cases, provide reassurance to parents, as no treatment is necessary. Live vaccines should be delayed until at least 11 months after IVIG, as antibodies in IVIG may reduce the effectiveness of these vaccines. Additionally, up to $1 \%-3 \%$ of patients may have recurrence of $\mathrm{KD}$ after recovering from the first episode.

\section{TAKE HOME MESSAGES}

1. $\mathrm{KD}$ is one of the most common types of vasculitis in childhood, predominantly affecting young children aged below five years; if left untreated, it increases the risk of coronary aneurysms as sequelae.

2. $\mathrm{KD}$ is a clinical diagnosis made in the presence of fever of at least five days and at least four out of five clinical criteria as per the $\mathrm{AHA}^{\prime}$ s recommendation (Box 1).

3. Patients with incomplete features should be further evaluated with blood and urine tests according to the AHA diagnostic algorithm (Fig. 1) for incomplete KD. There should be a high index of suspicion for incomplete KD.

4. BCG site inflammation and irritability, although not in the diagnostic criteria, are frequently encountered symptoms.

5. Differential diagnoses such as viral or bacterial infections, drug-related reactions and autoimmune disorders do not exclude KD. If fever persists despite adequate treatment, KD should be considered.

6. Early referral for workup for KD should be considered if any of the red flag symptoms are present (Box 2). 
7. Live vaccinations should be deferred for at least 11 months following IVIG administration.

\section{ACKNOWLEDGEMENTS}

We would like to thank A/Prof Thaschawee Arkachaisri, Head and Senior Consultant, KK Women's and Children's Hospital, Rheumatology and Immunology Service, Singapore, for his contribution of clinical pictures and his support for this article.

Four weeks later, Tom returned for another consultation. He was diagnosed with incomplete Kawasaki disease (KD) and was treated with intravenous immunoglobulin (IVIG) as an inpatient. He was kept on aspirin as his latest echocardiogram showed dilated coronary arteries. His mother was extremely anxious about the newonset skin peeling on his fingertips and toes. There was no recurrence of fever. On examination, Tom appeared happy and well. You reassured his mother that peeling is a natural progression of $K D$ and is usually self-limiting. You also took the opportunity to check Tom's health booklet and advised his mother that he was due for his pneumococcal booster vaccine, even though his vaccine for mumps, measles and rubella should be delayed for 11 months after IVIG treatment. You also encouraged his mother to get him vaccinated with the latest influenza vaccine.

\section{REFERENCES}

1. Uehara R, Belay ED. Epidemiology of Kawasaki disease in Asia, Europe, and the United States. J Epidemiol 2012; 22:79-85.

2. Gray H, Cornish J. Kawasaki disease: a need for earlier diagnosis and treatment. Arch Dis Child 2019; 104:615-6.

3. Dillon MJ, Eleftheriou D, Brogan PA. Medium-size-vessel vasculitis. Pediatr Nephol 2010; 25:1641-52.

4. Yeung RS. Kawasaki disease: update on pathogenesis. Curr Opin Rheumatol 2010; 22:551-60.

5. SingHealth. Kawasaki Disease in Children. Available at: https://www. healthxchange.sg/children/childhood-conditions/kawasaki-disease-children. Accessed June 10, 2020.

6. Newburger JW, Takahashi M, Burns JC, et al. The treatment of Kawasaki syndrome with intravenous gamma globulin. N Engl J Med 1986; 315:3417.

7. Kuo HC. Preventing coronary artery lesions in Kawasaki disease. Biomed J 2017; 40:141-6.

8. Tulloh RMR, Mayon-White R, Hamden A, et al. Kawasaki disease: a prospective population survey in the UK and Ireland from 2013 to 2015. Arch Dis Child 2019; 104:640-6. Erratum in: Arch Dis Child 2020;105:e5.

9. Son MBF, Newburger JW. Kawasaki disease. Pediatr Rev 2018; 39:78-90.

10. McCrindle BW, Rowley AH, Newburger JW, et al; American Heart Association Rheumatic Fever, Endocarditis, and Kawasaki Disease Committee of the Council on Cardiovascular Disease in the Young; Council on Cardiovascular and Stroke Nursing; Council on Cardiovascular Surgery and Anesthesia; and Council on Epidemiology and Prevention. Diagnosis, treatment, and long-term management of Kawasaki disease: a scientific statement for health professionals from the American Heart Association. Circulation 2017; 135:e927-99.

11. Moore A, Harnden A, Mayon-White R. Recognising Kawasaki disease in UK primary care: a descriptive study using Clinical Practice Research Datalink. $\mathrm{Br}$ J Gen Pract 2014; 64:e477-83.

12. Riphagen S, Gomez X, Gonzalez-Martinez C, Wilkinson N, Theocharis $P$. Hyperinflammatory shock in children during Covid-19 pandemic. Lancet 2020; 395:1607-8.

13. NYC Health. New York City Reports at Least 89 Cases of Multisystem Inflammatory Syndrome in Children (MIS-C). Available at: https://www1.nyc. gov/site/doh/about/press/pr2020/89-cases-of-mis-c-in-nyc.page. Accessed June 10, 2020 


\section{SINGAPORE MEDICAL COUNCIL CATEGORY 3B CME PROGRAMME} (Code SMJ 202101A)

1. Kawasaki disease (KD) is a common childhood vasculitis disorder and is now the most common cause of acquired heart disease in children in developed countries.

2. Untreated KD is associated with development of coronary artery aneurysms, putting patients at risk of myocardial ischaemia.

3. Patients with KD commonly present to primary care clinics for acute fever.

4. The most common cause of acute undifferentiated fever in children is KD.

5. There is a diagnostic test for KD.

6. Classical KD is diagnosed in the presence of fever of $\geq 5$ days, together with at least three of the five principal clinical features, including: (1) erythema and cracking of lips, strawberry tongue, and/or erythema of oral and pharyngeal mucosa; (2) bilateral suppurative conjunctivitis; (3) maculopapular, diffuse erythroderma or erythema multiforme-like rash; (4) erythema and oedema of the hands and feet in the acute phase and/or periungual desquamation in the subacute phase; (5) cervical lymphadenopathy $(\geq 1 \mathrm{~cm}$ in diameter).

7. Incomplete KD should be considered in any infant or child with prolonged unexplained fever and fewer than four of the principal clinical findings.

8. The diagnosis of KD should be considered in patients with persistent fever of $>5$ days and sterile pyuria.

9. Patients with persistent fever and cervical lymphadenopathy despite antibiotic treatment should be treated with a prolonged course of antibiotic therapy.

10. Bacille Calmette-Guérin vaccine site inflammation and irritability are common symptoms of KD.

11. COVID-19 infection is associated with multi-systemic inflammatory syndrome, which shares many characteristics with KD.

12. Patients with suspected KD can be managed as outpatients.

13. The presence of an infective aetiology such as infectious mononucleosis or scarlet fever excludes KD.

14. All clinical features of KD present simultaneously.

15. Patients who present with fever and one or two non-specific features such as rashes and non-suppurative conjunctivitis can be managed for viral fever even if the fever persists beyond five days.

16. First-line treatment for KD includes intravenous immunoglobulin (IVIG) and ibuprofen.

17. IVIG treatment should be given as early as possible within the first ten days of onset of fever, as soon as the diagnosis can be established, to reduce the risk of coronary aneurysm.

18. Patients with coronary artery aneurysm will be on long-term aspirin to reduce the risk of thrombosis and myocardial ischaemia.

19. All routine childhood vaccinations, including live vaccines, can be continued as usual after treatment with IVIG.

20. Urgent referral to a dermatologist is warranted if a patient with recent KD presents with peeling of the fingertips.

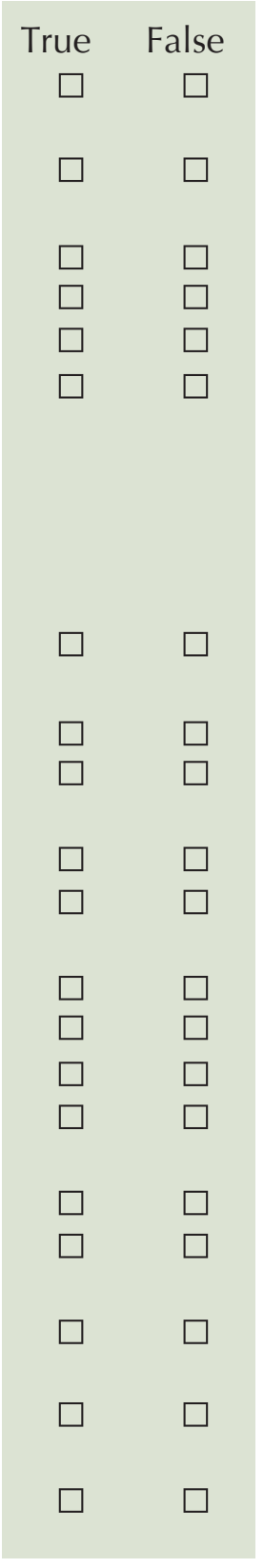

\section{Doctor's particulars:}

Name in full:

MCR no.:

Specialty:

Email:

\section{SUBMISSION INSTRUCTIONS:}

Visit the SMJ website: http://www.smj.org.sg/current-issue and select the appropriate quiz. You will be redirected to the SMA login page.

For SMA member: (1) Log in with your username and password (if you do not know your password, please click on 'Forgot your password?'). (2) Select your answers for each quiz and click 'Submit'.

For non-SMA member: (1) Create an SMJ CME account, or log in with your SMJ CME username and password (for returning users). (2) Make payment of SGD 21.40 (inclusive of $7 \%$ GST) via PayPal to access this month's quizzes. (3) Select your answers for each quiz and click 'Submit'.

RESULTS:

(1) Answers will be published online in the SMJ March 2021 issue. (2) The MCR numbers of successful candidates will be posted online at the SMJ website by 17 March 2021. (3) Passing mark is $60 \%$. No mark will be deducted for incorrect answers. (4) The SMJ editorial office will submit the list of successful candidates to the Singapore Medical Council. (5) One CME point is awarded for successful candidates. (6) SMC credits CME points according to the month of publication of the CME article (i.e. points awarded for a quiz published in the January 2021 issue will be credited for the month of January 2021, even if the deadline is in March 2021).

Deadline for submission (January 2021 SMJ 3B CME programme): 12 noon, 10 March 2021. 